\title{
Prevalência de anemia e deficiência de ferro: relação com índice de massa corporal em gestantes do Centro-Oes- te do Brasil*
}

\section{Prevalence of anemia and iron deficiency: association with body mass index in women of Brazilian Midwest.}

\author{
Rosângela M. S. de Camargo'1, Mariano M. Espinosa², Shirley F. Pereira³, Janine Schirmer ${ }^{4}$
}

\begin{abstract}
RESUMO
Objetivo: Verificar a prevalência de anemia e deficiência de ferro, analisar a correlação entre indicadores hematológicos e índice de massa corporal pré-gestacional/gestacional e o comportamento desses indicadores de acordo com a semana de gestação. Método: Estudo de corte transversal com 146 gestantes atendidas em ambulatório de pré-natal da rede pública de Cuiabá-MT, Centro-Oeste do Brasil, de maio de 2008 a maio de 2009. Os indicadores hematológicos foram coletados e relacionados entre si e com os índices de massa corporal. Analisou-se o comportamento dos indicadores hematológicos conforme a semana de gestação. O coeficiente de correlação de Pearson foi empregado para avaliar a associação entre as variáveis. Para estes testes, foi considerado o nível de significância menor que $5 \%$ ( $p<0,05)$. Resultados: A prevalência de anemia variou de 3,4\% a 4,8\%, considerando-se os valores para hemoglobina, hematócrito e volume corpuscular médio. Entretanto, as prevalências das alterações nos indicadores de reservas de ferro variaram de $11,0 \%$ para transferrina a $39,0 \%$ para ferritina. As correlações positivas estatisticamente significantes foram: hemoglobina e hematócrito $(0,85)$, ferro e índice de saturação de transferrina $(0,75)$. Observou-se comportamento constante no volume corpuscular médio, hematócrito e hemoglobina, e queda nestes indicadores ao final da 27ª semana de gestação. O IMC mostrou correlação negativa com o ferro sérico. Conclusão: Foi baixa a prevalência de anemia pelos níveis de hemoglobina, mas os indicadores de reservas de ferro mostraram maior prevalência de deficiência de ferro. Os indicadores de reservas de ferro que tiveram correlação mais forte entre si foram o ferro sérico e o índice de saturação da transferrina, sugerindo que sua combinação com outros indicadores hematológicos poderia melhor caracterizar a anemia por carência de ferro. O IMC e o ferro apresentaram correlação negativa.
\end{abstract}

Palavras-chave: Hematologia/Parâmetros. Gravidez. Índice de Massa Corporal. Cuidado Pré-Natal. Anemia.

* Artigo extraído da Tese de Doutorado "Consumo alimentar, estado nutricional de gestantes e indicadores de reservas de ferro" apresentada ao Programa de Pós-Graduação da Escola de Enfermagem da Universidade Federal de São Paulo (UNIFESP) em 2011.

${ }^{1}$ Doutora em Ciências, Professora Adjunta; ${ }^{2} \mathrm{PhD}$ em Estatística, Professor Associado; ${ }^{3}$ Doutora em Saúde e Ambiente, Professora Associada. Departamento de Alimentos e Nutrição da Universidade Federal de Mato Grosso.

${ }^{4}$ Doutora em Enfermagem Materno e Infantil, Professora Titular da Escola Paulista de Enfermagem da Universidade Federal de São Paulo.
Faculdade de Nutrição/Departamento de Alimentos e Nutrição. Campus Universitário. Bairro Boa Esperança Avenida Fernando Correa da Costa, № 2367 CEP-78060-900. Cuiabá/Mato-Grosso Fone/fax: (65)3615-8811. email: rosanms@terra.com.br

Artigo recebido em 24/09/2012 Aprovado para publicação em 06/03/2013 


\section{Introdução}

Anemia é uma situação caracterizada pela diminuição anormal da concentração de hemoglobina no sangue. É uma alteração comum que, geralmente, compromete as gestações e está relacionada, sobretudo, com a deficiência de alguns micronutrientes, entre eles o ferro, vitamina B12 e/ou ácido fólico, causada pelo aumento da exigência dos mesmos. ${ }^{1}$

A deficiência de ferro é o principal fator de risco para o desenvolvimento de anemias nutricionais. ${ }^{2}$ A carência de ferro ocorre no organismo, de forma gradual e progressiva, até que a anemia se manifeste. ${ }^{3}$ As gestantes representam um dos grupos populacionais mais vulneráveis às anemias nutricionais, em função da baixa ingestão durante esse período e aumento da demanda deste mineral. Como conseqüência associa-se a uma maior incidência de abortos, partos prematuros, baixo peso ao nascer e morte perinatal, entre outras. ${ }^{4}$

No diagnóstico da anemia gestacional, a hemoglobina sérica $<11 \mathrm{~g} / \mathrm{dl}$ tem sido usada com mais frequência nos serviços públicos de saúde de pré-natal, considerando sua praticidade e baixo custo. ${ }^{5}$ Entretanto, vários são os marcadores capazes de identificar os estoques de ferro no organismo, antes mesmo da diminuição dos níveis de hemoglobina. ${ }^{1}$ Estudos mostram que esses indicadores caracterizam de forma diferente a deficiência de ferro no organismo, variando conforme os pontos de corte utilizados como também em função do período da gestação. ${ }^{6,7,8}$

A gestação é caracterizada por ajustes fisiológicos e anatômicos que provocam mudanças importantes no organismo materno, incluindo os elementos do sangue circulante e o processo de expansão do volume plasmático com consequente hemodiluição. ${ }^{9}$

Em relação ao estado nutricional, sabe-se que as medidas antropométricas de peso e estatura são recomendadas no acompanhamento da gestação por sua fácil aplicabilidade e baixo custo. Da relação destas duas medidas, elevando-se a estatura ao quadrado, encontra-se o índice de massa corporal (IMC), que proporciona informações sobre as reservas energéticas e tem sido utilizado para avaliar o estado nutricional pré-gestacional e gestacional e para monitorar o ganho de peso durante a gestação. ${ }^{10}$

Dados da literatura vêm sugerindo a existência de associação entre estado nutricional avaliado através das medidas de peso, estatura e IMC e carências nutricionais específicas como anemia por deficiência de ferro. ${ }^{11,12}$
Estudo realizado com gestantes com a finalidade de avaliar a associação entre os níveis de ferritina, um dos indicadores das reservas de ferro, apontou correlação significante entre peso materno e ferritina sérica. ${ }^{13}$

Diante das mudanças hematológicas que ocorrem no organismo da gestante e a necessidade de diagnóstico e tratamento precoce da deficiência de ferro, com o intuito de contribuir para a promoção da saúde materna e fetal, este estudo teve como objetivo identificar a prevalência de anemia e deficiência de ferro em gestantes, analisar a correlação entre os índices hematológicos entre si e com os índices de massa corporal pré-gestacional e gestacional e verificar o comportamento desses índices de acordo com a idade gestacional em estudo.

\section{Método}

Trata-se de um estudo de corte transversal, realizado no ambulatório de pré-natal do Hospital Universitário Júlio Müller (HUJM) da Fundação Universidade Federal de Mato Grosso, localizado na cidade de Cuiabá, Estado de Mato Grosso. O HUJM é um hospital de referência e o ambulatório de assistência pré-natal atende gestantes, independente do risco gestacional.

A população de estudo foi constituída por todas as gestantes que compareceram no ambulatório de pré-natal, entre maio de 2008 e maio de 2009 . Os critérios para inclusão no estudo foram: gestantes com idade entre 19 e 49 anos de idade, gestação de feto único e estar no $2^{\circ}$ trimestre, entre a $14^{\mathrm{a}}$ e $28^{\mathrm{a}}$ semana. Foram excluídas as gestantes com enfermidades obstétricas e clínicas, exceto as anêmicas.

Foi considerado o $2^{\circ}$ trimestre de gestação, porque antes desse período é frequente a ocorrência de sintomas gastrointestinais, como náuseas, vômitos, dispepsia, pirose, ${ }^{14,15}$ os quais poderiam interferir na avaliação e também porque grande parte das gestantes procura a assistência pré-natal a partir deste período. ${ }^{16}$ A idade gestacional foi obtida com base nos resultados dos exames de ultrassonografia existentes no prontuário.

Os dados das gestantes foram obtidos por meio de entrevista realizada por duas nutricionistas treinadas, com a aplicação de um formulário que incluía questões sobre as condições socioeconômicas e história reprodutiva. Também foi coletado o sangue das gestantes em jejum para avaliação bioquímica.

As variáveis dependentes foram: hemoglobina 
(Hb), hematócrito (Hct) e volume corpuscular médio (VCM) e os indicadores de reservas de ferro: ferritina sérica (FS), ferro sérico ( $\mathrm{Fe})$, transferrina sérica (Transf.), capacidade total de ligação do ferro (CTFL) e índice de saturação da transferrina (IST).

As variáveis independentes consideradas foram: idade gestacional, índice de massa corporal prégestacional (IMCPG) e índice de massa corporal gestacional (IMCG).

Os exames bioquímicos foram realizados no laboratório de análises clínicas do HUJM e a coleta de sangue foi realizada após jejum de 10 horas, conforme as técnicas bioquímicas utilizadas na instituição.

A avaliação hematimétrica das gestantes, os métodos e equipamentos utilizados nas análises, os limites que definem as alterações e a classificação dos indicadores hematológicos estão descritos no Quadro 1.

O IMCPG foi estimado dividindo-se o peso pela estatura elevada ao quadrado, com base na estatura atual (em metros) e no peso pré-gestacional (em quilogramas) informado pela gestante, sendo utilizados como referências os limites propostos pelo Institute of Medicine dos Estados Unidos da América (IOM, $1990)^{22}$ : baixo peso para IMC $<19,8 \mathrm{~kg} / \mathrm{m}^{2}$; peso normal para IMC entre 19,8 e $26 \mathrm{~kg} / \mathrm{m}^{2}$; sobrepeso para o IMC, entre 26,1 e $29 \mathrm{~kg} / \mathrm{m}^{2}$ e obesidade para o IMC $>29 \mathrm{~kg} / \mathrm{m}^{2}$.

O IMCG foi obtido com base nas informações sobre a idade gestacional contidas no prontuário e nas

Quadro 1. Valores de referência e classificação dos índices hematológicos.

\begin{tabular}{|c|c|c|c|}
\hline $\begin{array}{c}\text { Índices } \\
\text { hematológicos }\end{array}$ & Método de análise & $\begin{array}{c}\text { Limites para } \\
\text { diagnóstico } \\
\text { de alteração }\end{array}$ & $\begin{array}{c}\text { Classificação } \\
\text { da condição } \\
\text { nutricional }\end{array}$ \\
\hline
\end{tabular}

Hemoglobina $(\mathrm{Hb})$

Hematócrito (Hct)

Volume Corpuscular Médio (VCM)

Ferro sérico $(\mathrm{Fe})$

Ferritina sérica (FS)

Transferrina (Transf.)

Capacidade Total de Ligação do Ferro (CTFL)

Índice de Saturação da Transferrina (IST)
Espectrofotometria com equipamento Pentra 80 Marca ABX, (Paris França)

Método de integração numérica do volume corpuscular médio (VCM)

Calculado diretamente a partir do histograma de RBC (Red Blood Cell) - Contagem de células vermelhas

Método colorimétrico automatizado (BT 3000, WIENER, (Biotécnica Instrumental, Roma, Itália)

Quimioluminescência (Modular E170 ROCHE, Alemanha, Frankfurt)

Método de nefelometria (processo de medida da concentração de uma emulsão por comparação de sua transparência com a de uma preparação padrão) (BT 3000, WIENER, (Biotécnica Instrumental, Roma, Itália)

Método colorimétrico automatizado (BT 3000 Marca WIENER, (Biotécnica Instrumental, Roma, Itália)

Calculado com a fórmula: IST $(\%)=$ Ferro Sérico / CTFL X 100
Abaixo de $11 \mathrm{~g} / \mathrm{dl}^{17}$

Anemia

Abaixo de $33 \% 18,19$

Anemia

Abaixo de $80 \mathrm{fl}^{18}$

Anemia

Abaixo de $67 \mu \mathrm{g} / \mathrm{dl}^{20}$

Deficiência de ferro

Abaixo de $22 \mu \mathrm{g} / \mathrm{dl}^{20}$

Deficiência de ferro

Acima de $400 \mu \mathrm{g} / \mathrm{dl}^{18} \quad$ Deficiência de ferro

Acima de $300 \mu \mathrm{g} / \mathrm{dl}^{14}$

Deficiência de ferro

Abaixo de $16 \%^{18,21}$

Deficiência de ferro 
medidas de peso e estatura coletadas no momento da entrevista. As gestantes foram pesadas em pé, descalças, com um mínimo de roupas e sem acessórios, sendo usada balança tipo plataforma, marca Filizola com variação de 100 gramas para obtenção da medida. A estatura foi tomada com estadiômetro Seca® com variação de $0,1 \mathrm{~cm}$, estando as gestantes descalças, com os pés unidos, em posição ereta, com a cabeça posicionada de acordo com o plano de Frankfurt. A leitura da estatura foi realizada no centímetro mais próximo. ${ }^{23}$

Para a classificação do estado nutricional gestacional, utilizou-se a recomendação do Ministério da Saúde, ${ }^{24}$ que classifica a gestante em baixo peso, peso normal, sobrepeso e obesidade, considerando-se o IMC e a idade gestacional por semana. ${ }^{25}$

A digitação e análise dos dados coletados na pesquisa foram realizadas no pacote estatístico SPSS versão 15.0 for Windows. Para a verificação da distribuição normal dos dados foi utilizado o teste estatístico de Shapiro. Também foram estimadas as médias, desvios padrão e construídos intervalos de confiança de $95 \%$ para as variáveis investigadas. O coeficiente de correlação linear (coeficiente de correlação de Pearson) foi calculado para avaliar a correlação entre as variáveis, considerando um nível de significância menor que $5 \%(\mathrm{p}<0,05)$.

O projeto foi aprovado sob $n^{\circ} 1468 / 2007$ pelo Comitê de Ética em pesquisa da Universidade Federal de São Paulo (UNIFESP) e sob n ${ }^{\circ}$ 384/2007 pelo Comitê de Ética em Pesquisa do Hospital Universitário Júlio Muller, obedecendo, portanto, aos princípios éticos da Resolução 196/96 do Conselho Nacional de Saúde. As gestantes que concordaram em participar do estudo assinaram o Termo de Consentimento Livre e Esclarecido.

\section{Resultados}

No período do estudo, 221 gestantes atenderam aos critérios de inclusão na pesquisa. Destas, 36 $(16 \%)$ constituíram perdas por não comparecerem à entrevista, restando 185. Destas 39 (21\%) foram perdas por não realizarem os exames bioquímicos, totalizando 146 gestantes participantes no estudo.

Para verificar se o grupo de 185 gestantes era homogêneo em relação ao grupo de 146 gestantes, foi realizada uma análise da variância múltipla, considerando a distribuição de probabilidade F. Dos resultados desta análise, verificou-se que não houve diferen- ça estatisticamente significante entre os grupos, considerando um nível de significância de 5\%, ou seja, a perda de 39 gestantes não interferiu na análise das 146 que permaneceram no estudo. Cabe observar que esta verificação também foi realizada utilizando a distribuição t de Student, considerando um nível de significância de 5\%, obviamente chegando aos mesmos resultados, uma vez que existem apenas dois grupos sendo comparados.

A média de idade das gestantes estudadas foi de 26,05 anos \pm 4,94, variando entre 19 e 41 anos. Entre elas, 64\% tinham mais de 8 anos de escolaridade. A média de renda familiar per capita foi de 1,6 salários mínimos, com 2,3 gestações, 0,44 abortos, 0,92 partos e realizaram 2,16 consultas de pré-natal, tendo relatado início do pré-natal com 12 semanas. As variáveis antropométricas mostraram que, em média, as gestantes tinham $159 \mathrm{~cm}$ de altura, IMCPG de 23,88 \pm 5,06 $\mathrm{kg} / \mathrm{m}^{2}$ e IMC gestacional de $26,18 \pm 5,17 \mathrm{~kg} / \mathrm{m}^{2}$.

Os dados da Tabela 1 mostram que as médias dos índices hematimétricos e dos indicadores séricos das reservas de ferro encontravam-se dentro dos limites da normalidade, exceto para transferrina e capacidade total de ligação do ferro. A prevalência de anemia variou de $3,4 \%$ a $4,8 \%$, tendo por referência os valores para $\mathrm{Hb}$, Hct e VCM. As prevalências das alterações nos indicadores das reservas de ferro foram mais elevadas, variando de $11,0 \%$ para transferrina a $39,0 \%$ para ferritina.

Os dados da Tabela 2 mostram a correlação entre as medidas do exame hematológico, utilizando o Coeficiente de Correlação de Pearson, estabelecendo-se o nível de significância em 5\% (p<0,05).

As correlações positivas estatisticamente significantes em ordem decrescente foram: hemoglobina e hematócrito $(0,85)$, ferro e índice de saturação de transferrina $(0,75)$; ferro e ferritina $(0,31)$; ferritina e índice de saturação de transferrina $(0,24)$ e transferrina e capacidade total de ligação do ferro $(0,20)$. As correlações negativas estatisticamente significantes em ordem decrescente foram: índice de saturação de transferrina e capacidade total de ligação de ferro ($0,57)$; ferritina e transferrina $(-0,40)$; ferritina e capacidade total de ligação do ferro $(-0,31)$; ferro e capacidade total de ligação do ferro $(-0,25)$ e ferro e transferrina $(-0,19)$.

A existência ou não de correlação entre as variáveis IMCPG e IMCG e os índices hematológicos está apresentada na Tabela 3 e as correlações foram consideradas em nível de significância de 5\% 
Tabela 1

Parâmetros hematológicos e de reservas de ferro de gestantes atendidas em ambulatório de pré-natal da rede pública de Cuiabá/MT, 2009.

\begin{tabular}{|c|c|c|c|c|c|c|}
\hline \multirow{2}{*}{$\begin{array}{l}\text { Parâmetros } \\
\text { hematológicos }\end{array}$} & \multirow[b]{2}{*}{$\mathbf{n}^{*}$} & \multirow[b]{2}{*}{ Média } & \multirow[b]{2}{*}{ DP } & \multirow[b]{2}{*}{ IC $\quad 95 \%$} & \multicolumn{2}{|c|}{$\begin{array}{l}\text { Prevalencia de anemıa e } \\
\text { de deficiência de ferro }\end{array}$} \\
\hline & & & & & $\mathbf{n}$ & $\%$ \\
\hline $\mathrm{Hb}(<11 \mathrm{~g} / \mathrm{dl})$ & 146 & 12,1 & 0,74 & $12,0-\quad 12,2$ & 7 & 4,8 \\
\hline $\operatorname{Hct}(<33 \%)$ & 146 & 36,1 & 2,27 & $35,8-36,5$ & 6 & 4,1 \\
\hline $\operatorname{VCM}(<80$ fl. $)$ & 146 & 89,2 & 4,89 & $88,4-\quad 89,9$ & 5 & 3,4 \\
\hline $\mathrm{Fe}(<67 \mu \mathrm{g} / \mathrm{l})$ & 143 & 86,0 & 31,5 & $80,7-\quad 91,2$ & 44 & 30,1 \\
\hline $\mathrm{FS}(<22 \mu \mathrm{g} / \mathrm{l})$ & 141 & 39,2 & 36,1 & $33,2-\quad 45,2$ & 57 & 39,0 \\
\hline Transf $(>400 \mu \mathrm{g} / \mathrm{dl})$ & 133 & 329,3 & 61,4 & $318,7-339,8$ & 16 & 11,0 \\
\hline IST $(<16 \%)$ & 142 & 29,7 & 14,0 & $27,3-\quad 32,0$ & 24 & 16,4 \\
\hline $\operatorname{CTLF}(>300 \mu \mathrm{g} / \mathrm{dl})$ & 142 & 273,3 & 77,5 & $260,5-286,2$ & 43 & 29,5 \\
\hline
\end{tabular}

* O valor de $\mathrm{n}$ dos indicadores hematológicos variou em função de alguns exames não terem sido realizados pelo laboratório, por problemas técnicos. DP; Desvio padrão. IC 95\%: Intervalo de confiança de $95 \%$.

\section{Tabela 2}

Coeficiente de correlação de Pearson entre as medidas do exame hematológico de gestantes atendidas em ambulatório de pré-natal da rede pública de Cuiabá/MT, 2009.

\begin{tabular}{|c|c|c|c|c|c|c|c|c|}
\hline $\begin{array}{c}\text { Índices } \\
\text { hematológicos }\end{array}$ & $\mathbf{H b}$ & Het & VCM & $\mathrm{Fe}$ & FS & Transf. & IST & CTFL \\
\hline $\mathrm{Hb}$ & 1 & & & & & & & \\
\hline $\mathrm{Htc}$ & 0,85 & & & & & & & \\
\hline $\mathrm{P}$ & $<0,001$ & & & & & & & \\
\hline $\mathrm{VCM}$ & 0,11 & 0,05 & & & & & & \\
\hline $\mathrm{P}$ & 0,192 & 0,548 & & & & & & \\
\hline $\mathrm{Fe}$ & 0,14 & 0,08 & 0,15 & & & & & \\
\hline $\mathrm{P}$ & 0,099 & 0,323 & 0,077 & & & & & \\
\hline FS & 0,07 & 0,07 & 0,08 & 0,31 & & & & \\
\hline $\mathrm{P}$ & 0,425 & 0,392 & 0,346 & $<0,001$ & & & & \\
\hline Transf & $-0,02$ & 0,04 & $-0,02$ & $-0,19$ & $-0,40$ & & & \\
\hline $\mathrm{P}$ & 0,810 & 0,653 & 0,785 & $\mathbf{0 , 0 2 9}$ & $<0,001$ & & & \\
\hline IST & $-0,03$ & $-0,05$ & 0,09 & $\mathbf{0 , 7 5}$ & 0,24 & $-0,11$ & & \\
\hline $\mathrm{p}$ & 0,710 & 0,590 & 0,266 & $<0,001$ & 0,005 & 0,214 & & \\
\hline CTFL & 0,07 & 0,07 & $-0,06$ & $-0,25$ & $-0,31$ & 0,20 & $-0,57$ & 1 \\
\hline $\mathrm{p}$ & 0,401 & 0,437 & 0,461 & 0,003 & $<0,001$ & 0,021 & $<0,001$ & \\
\hline
\end{tabular}


$(\mathrm{p}<0,05)$. Não foram observadas correlações positivas estatisticamente significantes. As correlações negativas estatisticamente significantes em ordem decrescente foram: IMC gestacional e ferro $(-0,23)$, IMC gestacional/volume corpuscular médio $(-0,20)$ e IMC pré-gestacional/ferro $(-0,19)$.

Neste estudo, a diferença das médias do IMC pré-gestacional $\left(23,88 \mathrm{~kg} / \mathrm{m}^{2}\right)$ e do IMC gestacional $\left(26,18 \mathrm{~kg} / \mathrm{m}^{2}\right)$ para um IC $95 \%(1,98 ; 2,61)$ foi de 2,30 $\mathrm{kg} / \mathrm{m}^{2}$, mostrando que os dois limites do intervalo são positivos, o que indica que, em média, o IMC aumentou de maneira estatisticamente significante.
Na Figura 1, observa-se o comportamento da média dos índices hematimétricos e nota-se comportamento constante no volume corpuscular médio, hematócrito e hemoglobina e queda nestes marcadores, ao final da $27^{\mathrm{a}}$ semana de gestação.

A média dos índices das reservas de ferro em relação à idade gestacional por semana são mostrados na Figura 2 e verifica-se que o comportamento irregular foi maior para os índices de transferrina e capacidade de ligação do ferro, mantendo-se quase constante para o ferro, ferritina e índice de saturação da transferrina.

\section{Tabela 3}

Coeficiente de correlação de Pearson entre os índices hematológicos e o IMC pré-gestacional e gestacional de gestantes atendidas em ambulatório de pré-natal da rede pública de Cuiabá/MT, 2009.

\begin{tabular}{lcc}
\hline Índices hematológicos & IMC pré-gestacional & IMC gestacional \\
\hline Hemoglobina & $-0,025(\mathrm{p}=0,764)$ & $-0,004(\mathrm{p}=0,958)$ \\
Hematócrito & $0,059(\mathrm{p}=0,476)$ & $0,048(\mathrm{p}=0,563)$ \\
Volume corpuscular médio & $-0,138(\mathrm{p}=0,098)$ & $\mathbf{- 0 , 1 9 6}(\mathbf{p}=\mathbf{0 , 0 1 8})$ \\
Ferro & $\mathbf{- 0 , 1 8 7}(\mathbf{p}=\mathbf{0 , 0 2 5})$ & $\mathbf{- 0 , 2 2 8}(\mathbf{p}=\mathbf{0 , 0 0 6})$ \\
Ferritina & $0,025(\mathrm{p}=0,768)$ & $-0,020(\mathrm{p}=0,816)$ \\
Transferrina & $-0,044(\mathrm{p}=0,619)$ & $-0,025(\mathrm{p}=0,778)$ \\
Índice de saturação da transferrina & $-0,127(\mathrm{p}=0,131)$ & $-0,142(\mathrm{p}=0,091)$ \\
Capacidade total de ligação do ferro & $-0,002(\mathrm{p}=0,984)$ & $0,030(\mathrm{p}=0,723)$ \\
\hline
\end{tabular}

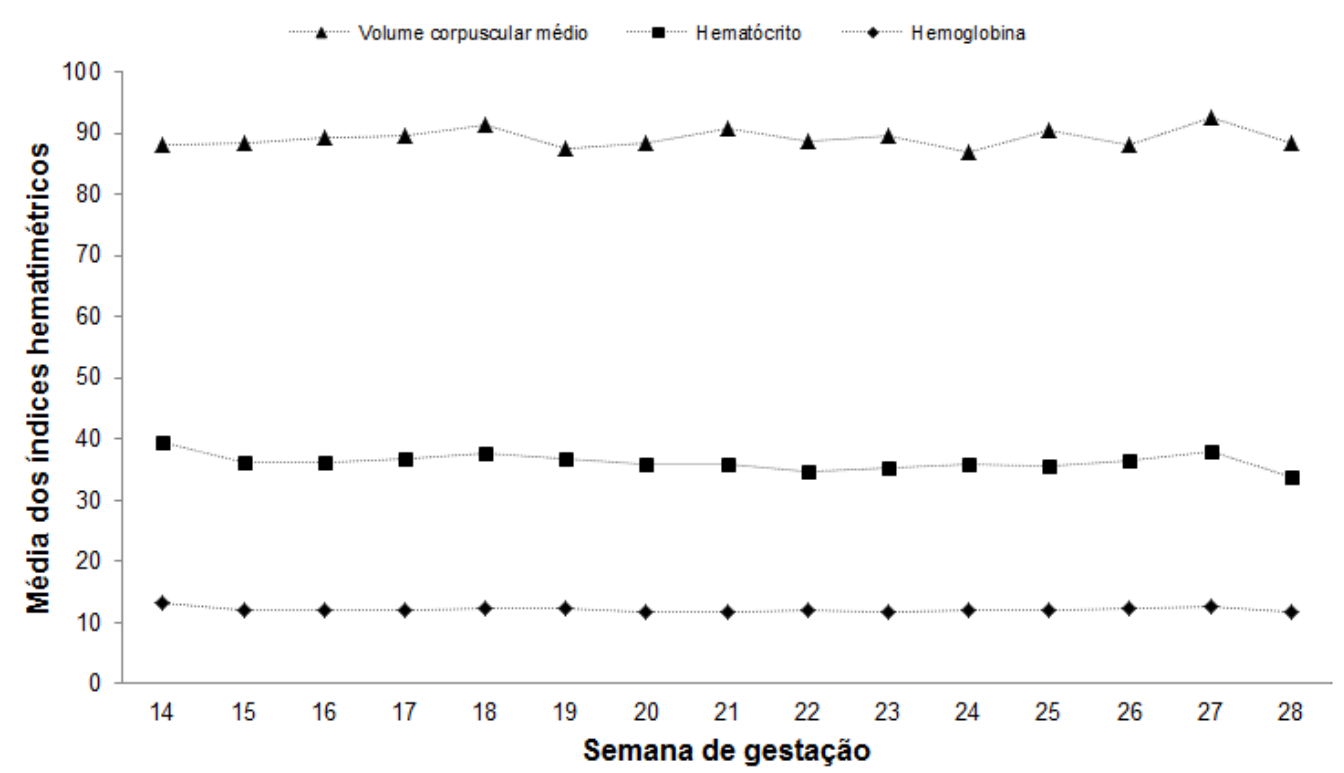

Figura 1. Gráfico das médias dos indicadores hematimétricos e semana de gestação. 


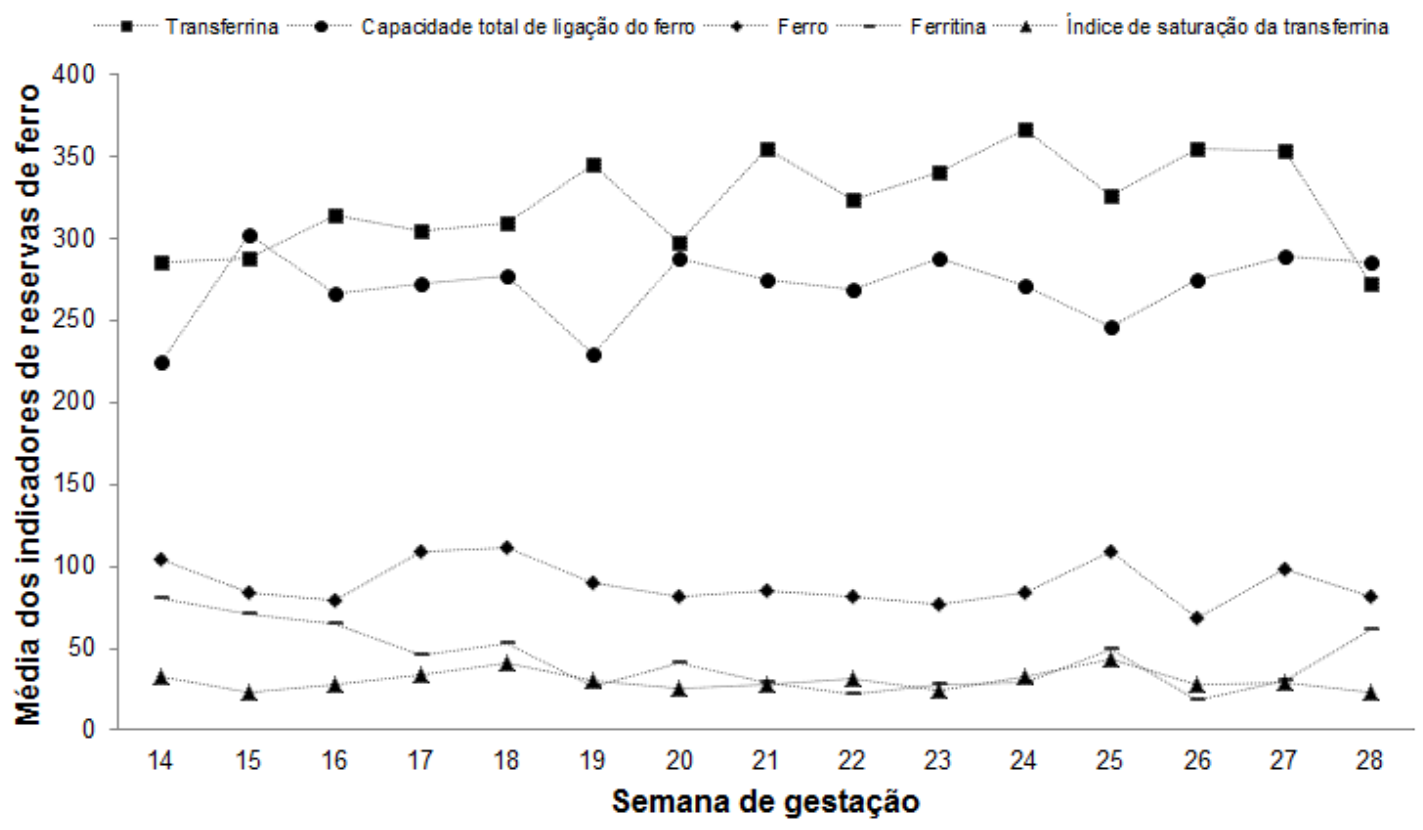

Figura 2. Gráfico das médias dos indicadores de reserva de ferro e semana de gestação.

\section{Discussăo}

Neste estudo, verificou-se que foi baixa a prevalência de anemia, de acordo com os níveis de hemoglobina, se comparado com outros estudos, ${ }^{19,24}$ entretanto os indicadores de reservas de ferro foram mais eficazes para detectar a deficiência de ferro.

Estudo utilizando a ferritina para avaliar a deficiência de ferro, aponta que esta geralmente se instala antes mesmo que os valores de hemoglobina se apresentem reduzidos e que são vários os indicadores empregados para identificar a deficiência de ferro em gestantes. ${ }^{1}$

Conforme alguns estudos, ${ }^{1,5}$ a hemoglobina é o único parâmetro rotineiramente utilizado na rede pública de assistência pré-natal, o que sugere que a maioria das gestantes com deficiência de ferro não é identificada. Por essa razão tem sido proposto que a hemoglobina deve estar associada a outros indicadores, para melhor identificar a deficiência desse nutriente tão importante, sobretudo na gestação.

$\mathrm{Na}$ avaliação do coeficiente de correlação entre os indicadores hematológicos, constatou-se que a maior correlação foi entre hemoglobina e hematócrito $(0,85)$. A pesquisa de Massucheti et al. $(2009)^{4}$ também constatou correlação linear forte entre estas duas variáveis, mostrando que as gestantes que apresentavam hemoglobina sérica $<11 \mathrm{~g} / \mathrm{dl}$, também tinham va- lores de hematócrito inferiores a 33\%. Os autores destacaram a importância desses indicadores na gestação, considerando que são usados com frequência como teste de triagem e confirmação de diagnóstico de anemia.

Verificou-se também forte correlação positiva estatisticamente significante entre ferro sérico e o índice de saturação da transferrina $(0,75)$. A utilização isolada do indicador de ferro sérico tem pouco uso clínico, pois não reflete realmente a depleção de ferro, além de apresentar consideráveis variações, em um mesmo dia, em indivíduos normais. Além disso, baixas concentrações de ferro são encontradas não apenas em pacientes com anemia ferropriva, mas também como consequência de infecção e doenças crônicas. ${ }^{20}$

Por sua vez, o índice de saturação da transferrina tem seu valor dependente da concentração de ferro e transferrina. Desse modo, sua associação com o ferro sérico pode ser considerada útil na identificação de pacientes com depleção dos estoques de ferro, uma vez que sua alteração é mais precoce que a diminuição dos níveis de hemoglobina, possibilitando assim a identificação da deficiência de ferro antes do surgimento da anemia, último estágio da carência de ferro.

Neste estudo, considerando o estado nutricional de ferro pela ferritina e ferro sérico verificou-se fraca correlação positiva significante $(0,31)$. Estudo realizado com gestantes adolescentes não identificou pa- 
cientes com depleção dos estoques de ferro pela ferritina sérica $(<12 \mathrm{~g} / \mathrm{dl})$, embora o ponto de corte utilizado tenha sido inferior ao empregado neste estudo, e todas as gestantes adolescentes apresentavam estoques de ferro dentro da normalidade $(>50 \mathrm{mg} / \mathrm{dl}) .{ }^{20}$

A ferritina sérica vem sendo considerada o melhor indicador para detectar a deficiência de ferro, entretanto pode se apresentar elevada nas doenças inflamatórias agudas e crônicas, abuso de álcool e câncer, sobretudo nos países onde as doenças crônicas e endêmicas são frequentes..$^{21,26}$

Os resultados deste estudo mostraram que dos parâmetros do estado nutricional de ferro analisados que mais se correlacionaram entre si para caracterizar a natureza da anemia foram o ferro e o índice de saturação da transferrina, seguidos do ferro e a ferritina, sugerindo que a combinação destes indicadores poderia identificar a deficiência de ferro, antes mesmo da redução dos níveis de hemoglobina.

Em relação à existência, ou não, de correlação entre as variáveis IMC pré-gestacional e gestacional e os índices hematológicos, não foram observadas correlações positivas estatisticamente significantes. Destacou-se correlação negativa estatisticamente significante entre IMCPG e IMCG e ferro sérico $(-0,19$ e $0,23)$, o que sugere que quanto maior o IMC, menor será o nível de ferro sérico.

Estudo analisando a associação entre estado nutricional pré-gestacional e os desfechos maternos e do concepto não encontrou associação entre estado nutricional pelo IMC pré-gestacional e anemia materna, de acordo com os níveis de hemoglobina11. Por outro lado, autores constataram que quanto maior o IMC na primeira consulta maior foi a concentração média de hemoglobina. ${ }^{27}$

Conforme o IOM (1990)22, a faixa de IMC prégestacional considerada normal para a gestante encontra-se entre 19,8 e $26 \mathrm{~kg} / \mathrm{m}^{2}$. Os resultados obtidos neste estudo apontaram, em média, IMC de 23,88 $5,06 \mathrm{~kg} / \mathrm{m}^{2}$, evidenciando normalidade para situação nutricional. Entretanto, destaca-se a ocorrência de variação mínima de $16,23 \mathrm{~kg} / \mathrm{m}^{2}$ e máxima de 42,60 $\mathrm{kg} / \mathrm{m}^{2}$, indicando a presença de baixo peso e obesidade no grupo de gestantes em estudo.

Pesquisa mostra que a transição nutricional que se desenvolve no Brasil evidencia a ocorrência simultânea e o crescimento de dois problemas de natureza oposta: por um lado, o sobrepeso/obesidade, condições típicas dos excessos alimentares e por outro as anemias carenciais, resultado da carência nutricional. ${ }^{28}$
É complexo determinar os fatores nutricionais associados à variação nos índices hematimétricos e estado nutricional de ferro diante das diferentes metodologias empregadas para classificação do estado nutricional pelo IMC. Somam-se ainda a estas limitações a carência de estudos encontrados na literatura relacionando o IMC com os marcadores hematológicos.

Estes resultados reforçam a necessidade da realização de mais pesquisas sobre a avaliação do estado nutricional pelo IMC pré-gestacional e gestacional, não só com ênfase nos desvios ponderais, mas também nos conhecimentos sobre os marcadores dos micronutrientes para o combate às carências nutricionais de ferro, ${ }^{6}$ fato também observado em relação ao período gestacional em estudo ( $14^{\mathrm{a}}$ a $28^{\mathrm{a}}$ semanas).

Em relação ao comportamento dos indicadores de reservas de ferro com a progressão da idade gestacional, estudo demonstrou que as deficiências de ferritina e ferro sérico também aumentam com a evolução da semana de gestação $\left(16,28\right.$ e $36^{a}$ semanas $) .{ }^{29}$ Desse modo, acredita-se que esses achados também possam estar relacionados com a hemodiluição fisiológica que ocorre na gravidez.

Neste trabalho, verificou-se que os níveis de todos os indicadores das reservas de ferro, embora tenham apresentado grande oscilação da $14^{\mathrm{a}}$ até a $28^{\mathrm{a}}$ semana de gestação, diminuíram ao final do $2^{\circ}$ trimestre, com exceção da ferritina que aumentou. Resultados semelhantes são mostrados em estudo realizado com gestantes fazendo uso de diferentes doses de suplemento ferroso a partir de $18^{\mathrm{a}}$ semana de gestação até a $8^{\mathrm{a}}$ semana pós-parto com objetivo de avaliar os efeitos colaterais da suplementação com ferro, que constatou aumento dos níveis de ferritina por volta da $32^{\mathrm{a}}$ semana de gestação, independente das diferentes doses de suplemento ferroso. ${ }^{26}$

Este estudo de corte transversal apresenta limitações, recomendando prudência na interpretação dos resultados. Portanto, são necessárias pesquisas que investiguem, de forma mais aprofundada, as reservas de ferro do organismo materno e sua relação com o estado nutricional, especialmente abordando o contexto em que as escolhas alimentares ocorrem, de modo a contribuir para o entendimento de questão tão complexa, multifatorial e paradoxal como a ocorrência simultânea de anemia por deficiência de ferro e obesidade e assim, subsidiar propostas de intervenção tendo em vista a promoção da alimentação saudável nesse grupo da população. 


\section{Conclusão}

Os achados desta pesquisa mostraram baixa prevalência de anemia pelos níveis de hemoglobina, entretanto, os indicadores de reservas de ferro mostraram maior prevalência de deficiência deste mineral.

Os indicadores hematimétricos que tiveram correlação mais forte entre si, foram a hemoglobina e o hematócrito e, entre os marcadores das reservas de ferro, o ferro sérico e o índice de saturação da trans- ferrina. Desse modo, sugere-se que a combinação entre estes indicadores hematológicos e de reserva de ferro poderia melhor caracterizar a anemia por carência de ferro.

Não foi observada correlação positiva significante entre os índices hematológicos e os de massa corporal pré-gestacional e gestacional. Houve correlação negativa estatisticamente significante entre os índices de massa corporal pré-gestacional e gestacional e ferro sérico.

\begin{abstract}
Objective: To investigate the prevalence of anemia and iron deficiency, to analyze the correlation of hematological indices with pre-gestational / gestational body mass, and the behavior of these indices according to the gestational age. Method: Cross-sectional study with 146 pregnant women attending public prenatal care service in Cuiabá city, state of Mato Grosso, Midwest of Brazil, from May 2008 to May 2009. Hematological indices were collected and related to each other and with the body mass index. The behavior of hematological indices was analyzed according to the gestational age. Pearson's coefficient correlation was used to assess association between variables. For these tests, it was considered the significance level of $5 \%(p<0.05)$. Results: The prevalence of anemia ranged from $3.4 \%$ to $4.8 \%$, considering the values for hemoglobin, hematocrit and mean corpuscular volume. However, the prevalence of changes in iron stores indices ranged from $11.0 \%$ for transferrin to $39.0 \%$ for ferritin. Statistically significant positive correlations were: hemoglobin and hematocrit $(0.85)$, iron and transferrin saturation index (0.75). It was observed a constant mean corpuscular volume, hematocrit and hemoglobin, and decrease in these indices in the end of 27 weeks of gestation. BMI showed negative correlation with serum iron. Conclusion: The prevalence of anemia by hemoglobin levels was low, but indicators of iron stores showed higher prevalence of iron deficiency. Among the iron stores indexes were more strongly correlated with each other were serum iron and transferrin saturation index, suggesting that their combination with other hematological indices could better characterize the iron deficiency anemia. The BMI and iron were negatively correlated.
\end{abstract}

Key-words: Parameters/ Hematology. Pregnancy. Body Mass Index. Prenatal Care. Anemia.

\section{Referências}

1. Dani C, Rossetto S, Castro SM, Wagner SC. Prevalência da anemia e deficiências nutricionais, através de diferentes parâmetros laboratoriais, em mulheres grávidas atendidas em dois serviços de saúde pública no Rio Grande do Sul. Rev Bras Anal Clin. 2008; 40: 171-5.

2. World Health Organization and Centers for Disease Control and Prevention. Assessing the iron status of populations: Report of a Joint World Health Organization, Centers for Disease Control and Prevention Technical Consultation on the assessment of Iron status at the Population Leve. Geneva; 2004.

3. Paiva AA, Rondo PHC, Latorre MRDO, Cardoso MAA, Gondim SSR. Relationship between the iron status of pregnant women and their newborns. Rev Saúde Pública 2007; 41: 321-7.

4. Massucheti L, Corso ACT, Moreira EAM. Prevalência de anemia em gestantes atendidas na rede pública de saúde do município de Florianópolis (SC). Cad Saúde Coletiva 2009; 10 : 417-31.

5. Dal Pizzol TS, Giugliani ERJ, Mengue SS. Associação entre o uso de sais de ferro durante a gestação e nascimento prétermo, baixo peso ao nascer e muito baixo peso ao nascer. Cad Saúde Pública 2009; 25: 160-8.

6. Milman N. Prepartum anaemia: prevention and treatment. Ann Hematol 2008; 87: 949-59.

7. Totti HKSB, Zimmermmann JB, Pena DMF, Pereira MP, Bittencourt $C$, Coutinho T. Frequência de anemia e valores de normalidade para hemoglobina em gestantes. HU Rev. Juiz de Fora 2010; 35: 282-6.

8. Szafarc, SC. Políticas públicas para o controle da anemia ferropriva. Rev Bras Hematol Hemoter 2010; 32 Supl.2: 2-7.

9. Gamboa LV, Duarte RQ, Martinez PG, Castilçho GV. Prevalencia de anemia ferropénica en mujeres embarazadas rurales en Valladolid, Yucatán, México. Ginecol Obstet Mex 2009; 77: 544-9. 
10. Kac G, Velasquez-Melendez G. Ganho de peso gestacional e macrossomia em uma coorte de mães e filhos. J Pediatr 2005; 81: 47-53.

11. Padilha PC, Saunders C, Machado RCM, Silva CL, Bull A, Sally EOF, Accioly E. Associação entre o estado nutricional prégestacional e a predição do risco de intercorrências gestacionais. Rev Bras Ginecol Obstet 2007; 29: 511-8.

12. Vasconcelos IAL, Côrtes MH, Coitinho DC. Alimentos sujeitos à fortificação compulsória com ferro: um estudo com gestantes. Rev Nutr 2008; 21: 149-60.

13. Vazirinejad R, Esmaeili A, Vazirinajad H, Hassanshahi G. Ferritin concentration and pregnancy outcome: linear models for predicting birthweight and birth length. Food Nutr Bull 2007; 28: 419-25.

14. Vitolo MR. Da gestação à adolescência. Rio de Janeiro: Reichmann \& Autores Editores, 2003.

15. Malta MB, Carvalhares MABL, Parada CMGL, Corrente JE. Utilização das recomendações de nutrientes para estimar prevalência de consumo insuficiente das vitaminas $\mathrm{C}$ e E em gestantes. Rev Bras Epidemiol 2008; 11: 573-83.

16. Cortês $\mathrm{MH}$, Vasconcelos IAL, Coitinho DC. Prevalência de anemia ferropriva em gestantes brasileiras: uma revisão dos últimos 40 anos. Rev Nutr 2009; 22: 1-11.

17. World Health Organization. Iron deficiency anaemia: assessment, prevention, and control: a guide for programme managers. Geneve: The Organization; 2001.

18. Gabrielloni MC. Estudo da prevalência de anemia e da perda hemática em parto vaginal e cesárea (tese). São Paulo: UNIFESP/EPM: 2002.

19. Piammongkol S, Chongsuvivatwong V, Williams G, Pornpatkul $M$. The prevalence and determinants of iron deficiency anemia in rural thai-muslim pregnant women in pattani province. Southeast Asian J Med Public Health 2006 1; 37: 553-7.
20. Papa EAC. A anemia por deficiência de ferro e sua absorção em gestantes adolescentes (tese). São Paulo: UNIFESP/EPM: 2002.

21. Soares NN, Mattar R, Camano L, Torloni MR. Iron deficiency anemia and iron stores in adult and adolescent women in pregnancy. Acta Obstetr Gynecol 2010; 89: 343-9.

22. Institute of Medicine. Nutrition during pregnancy. Washington: National Academy Press; 1990.

23. Cuppari L. Guia de Nutrição. Nutrição Clínica do adulto. $2^{\underline{a}}$ ed. São Paulo: Ed Manole Ltda, 2005

24. Brasil. Ministério da Saúde. Pré-natal e puerpério: atenção qualificada e humanizada. Manual técnico. Brasília: Ministério da Saúde; 2005.

25. Atalah E, Castilho C, Castro R, Aldea A. Propuesta de um nuevo estándar de evaluación nutricional en embarazadas. Rev Méd Chile 1997; 125:1429-36.

26. Milman N. Iron prophylaxis in pregnancy - general or individual and in which dose? Ann Hematol 2006; 85: 821-8.

27. Sato APS, Fujimori E, Szarfarc SC, Borges ALV, Tsunechiro MA. Consumo alimentar e ingestão de ferro de gestantes e mulheres em idade reprodutiva. Rev Latino-Am Enf 2010; 18: 113-21.

28. Batista Filho M, Souza AI, Miglioli TC, Santos MC. Anemia e obesidade: um paradoxo da transição nutricional brasileira. Cad Saúde Pública 2008; 24(Supl 2): S247-57.

29. Cruz RD. A avaliação da deficiência de ferro durante o processo gestacional e sua relação com o consumo alimentar e a suplementação com ferro (dissertação). São Paulo: Fac Ciênc Farmac USP: 2009. 